\title{
Valoración del paisaje urbano- ambiental de Juriquilla y Santa Rosa Jáuregui, Querétaro, México
}

\section{Evaluating the urban environmental landscape of Juriquilla and Santa Rosa Jauregui, Queretaro, Mexico}

\author{
Juan Alfredo Hernández Guerrero*
}

\begin{abstract}
Cities are composed of dynamic and heterogeneous landscapes of complex evaluation and dissimilar urban and environmental management. To analyze this complexity, the quality of the urban-environmental landscape of Juriquilla and Santa Rosa Jauregui in the city of Queretaro was evaluated. Video recording of the landscape, urban and environmental indicators, inverse distance interpolation and multi-criteria evaluation were used. In the results, Santa Rosa Jauregui presented low quality of the urban-environmental landscape and priority attention zones were identified, whereas in Juriquilla the quality was high with intermediate and monitoring zones. It concludes with a replicable and support proposal in territorial planning.
\end{abstract}

Keywords: evaluation, quality, landscape, urban, environmental.

\section{Resumen}

Las ciudades están compuestas por paisajes dinámicos y heterogéneos de compleja valoración y disímil gestión urbana y ambiental. Para analizar esa complejidad, se valoró la calidad del paisaje urbano-ambiental de Juriquilla y Santa Rosa Jáuregui en la ciudad de Querétaro. Se emplearon videograbaciones del paisaje, indicadores urbanos y ambientales, interpolación de distancia inversa y evaluación multicriterio. En los resultados, Santa Rosa Jáuregui presentó baja calidad del paisaje urbano-ambiental y se identificaron zonas de atención prioritarias; en Juriquilla la calidad fue alta con zonas intermedias y de monitoreo. Se concluye con una propuesta replicable y de apoyo para la planeación territorial.

Palabras clave: valoración, calidad, paisaje, urbano, ambiental.

*Universidad Autónoma de Querétaro, correo-e: juan.hernandez@uaq.mx 


\section{Introducción}

Al comenzar el siglo XX, disciplinas como la antropología, la sociología y la geografía tomaron el paisaje como unidad de análisis para interpretar porciones dinámicas del espacio, a través de relaciones convolutivas entre lo biofísico y lo cultural (Maderuelo, 2005; Gailing y Leibenath, 2015). Esas disciplinas mencionan que el paisaje (según el lugar, cultura e identidad) se explica a través de interacciones, modificaciones y relaciones sociales y naturales, en la ocupación, transformación e interpretación de fracciones del espacio subjetivo sentido y vivido (Fürstenau, 2009; Urquijo y Barrera-Bassols, 2009; Medina, 2010; Urquijo y Bocco, 2011; Folch y Bru, 2017).

El paisaje urbano se define como la relación de rasgos visibles de las interacciones biofísicas y culturales que interactúan dentro de entornos artificialmente construidos (Maderuelo, 2010; Lalana, 2011). Al mismo tiempo, los estilos de vida, actividades y necesidades humanas producen ajustes y transformaciones que recrean paisajes diferenciados por las condiciones del entorno inmediato (Matsuoka y Kaplan, 2008; Wu, 2010; Taylor, 2016; Liu et al., 2017).

La ciudad contiene múltiples entornos que denotan paisajes heterogéneos diferenciados por sectores (habitacional, comercial, industrial, recreativo), compuestos de variables urbanas (equipamiento, infraestructura, cobertura, estructura) y ambientales (vegetación, ríos, temperatura, contaminación) (Andersson, 2006; Matsuoka y Kaplan, 2008; Hernández-Guerrero, 2015); dichas variables, al analizarse integralmente, pueden ser una alternativa de gestión en la planeación urbana y territorial; su valoración puede incluir interpretaciones cualitativas y cuantitativas para diferenciar entornos según su estructura, condición, organización, componentes, uso y manejo del fondo escénico (Pérez, 2000; Pasimeni et al., 2012; Gavrilidis et al., 2016; Bürgi et al., 2017).

La comprensión del paisaje urbano y ambiental, al no ser lineal, está supeditada a constructos mentales, sensaciones estéticas y sentimientos afectivos, que a su vez producen asociaciones de la interpretación del entorno inmediato (Galindo y Corraliza, 2000; Maderuelo, 2010; Lalana, 2011; Ellard, 2015).

En ese sentido destacan los métodos directos para valorar el paisaje mediante técnicas subjetivas en las que se emplean escalas de rango o de orden, a través de la contemplación parcial o total del paisaje, sea in situ o a través de fotografías, imágenes digitales (tabletas o computadoras) y videograbaciones; ese tipo de valoración debe contener criterios claros de la diversidad de elementos que incluyen los paisajes, de lo contrario, el juicio del evaluador puede aumentar o disminuir el valor de la respuesta; por esta 
razón, también se requieren evaluadores capacitados en la técnica (MuñozPedreros, 2004).

Los métodos directos de valoración del paisaje con enfoque cultural, histórico, arquitectónico, ambiental, urbano o económico comúnmente son utilizados en el análisis de las ciudades, pues las técnicas permiten identificar, diferenciar y evaluar los múltiples entornos artificialmente construidos que las integran, así como la configuración y reconfiguración de su dinámica evolutiva (Cabrerizo, 2013; Liu et al., 2017; AguileraBenavente et al., 2011; Hofmann et al., 2012).

El presente trabajo emplea un enfoque de análisis de paisaje urbanoambiental que considera la calidad visual de éste, a través de técnicas estético-perceptuales (Muñoz-Pedreros, 2004); se utilizan elementos urbanísticos como edificaciones, infraestructura, vialidades, mobiliario y espacios abiertos, así como elementos ambientales, sea contaminación, vegetación, confort térmico y acústico (Briceño y Gil, 2003; Matsuoka y Kaplan, 2008; Briceño et al., 2011; Keshtkaran et al., 2017; Barrasa, 2013; Liu et al., 2017; Hernández-Guerrero, 2015; Hernández-Guerrero y Osorno, 2018).

La técnica comprende el análisis de indicadores cualitativos-cuantitativos (realizados por expertos) resultantes de videograbaciones obtenidas en recorridos in situ (Karmanov y Hamel, 2008), con ello se interpretó y diferenció la calidad del paisaje urbano y ambiental.

Con el fin de facilitar la manipulación de información, transformación de datos y representación, el proceso se acompañó del método de evaluación multicriterio a través de sistemas de información geográfica (SIG), lo cual es útil para la relación de indicadores y análisis espacial que, en este caso, fue a través de la superposición de capas por indicadores, estandarización y ponderación, para obtener resultados ágiles, detallados y escenarios alternativos (Buzai, 2015).

Por otra parte, cabe decir que en las ciudades de países en desarrollo la valoración ambiental es escasa, ya que suele apoyarse de indicadores obtenidos con costosos equipos de medición y la valoración urbana suele carecer de bases de datos confiables y monitoreo (Pérez, 2000; Nogué, 2010; Gavrilidis et al., 2016; Guzmán et al., 2017; Nogué et al., 2019). En este caso, los resultados de la valoración de la calidad del paisaje urbanoambiental incluyen bases de datos, productos cartográficos y modelos de fenómenos espaciales que ayudan en la toma de decisiones, sea en la identificación de sitios prioritarios, determinación de instrumentos de medición ambiental y acompańamiento en las estrategias de planeación urbana y ambiental (Hernández-Guerrero y Osorno, 2018; Nogué et al., 2019).

Con base en lo anterior, cobra mayor relevancia la valoración en ciudades con acelerada urbanización, ya que se obtendría una mejor 
valoración del paisaje urbano-ambiental debido a la dinámica y reconfiguración del paisaje.

En México, 12 de las principales ciudades producen entornos urbanos disímiles asociados a su crecimiento poblacional, expansión física y dinamismo económico, comercial e industrial; en la zona norte, Tijuana, Mexicali, Monterrey, San Luis Potosí y Zacatecas; en la zona centro, Aguascalientes, León, Querétaro y Tlaxcala, y en la zona sur, Mérida y Tuxtla Gutiérrez. En esas ciudades la tasa de crecimiento promedio anual supera el 2\% (frente a la tasa nacional anual de 1.37\%); además, suman un total de 13,940,080 habitantes y cuentan con una densidad promedio de $696.3 \mathrm{hab} / \mathrm{km}^{2}$ (Jusidman et al., 2016; Inegi, 2017).

De las anteriores destaca la ciudad de Querétaro, pues desde 1960 sus problemas urbanos y ambientales son acompañados por desarrollo industrial, habitacional y comercial (Hernández-Guerrero et al., 2016). En los últimos 25 años, la ciudad presenció un importante crecimiento demográfico y físico: pasó de tener 552,470 habitantes en 1990 a 1,255,185 habitantes en 2015, mientras que su superficie construida aumentó de 6492 hectáreas a 18,216 hectáreas (Inegi, 1990 y 2015a; Göbel, 2015). A finales del año 2015 la ciudad registraba la llegada de 67 personas por día: 24,400 personas al año (Hernández-Guerrero et al., 2016). También, entre 2016 y 2017 la industria generó 38\% del PIB de la entidad y produjo 22,000 empleos al año (Godínez y Alvarez-Castañon, 2017).

El norte de la ciudad de Querétaro es una de las regiones con mayor dinámica urbana, destacan Juriquilla y Santa Rosa Jáuregui por la multiplicidad de actividades con repercusiones en la reconfiguración territorial y formación de entornos fragmentados, cambios de uso de suelo, ocupación de lugares susceptibles a riesgo, degradación y contaminación ambiental (PNUMA, 2008; Göbel, 2015; Hernández-Guerrero et al., 2016). Ambos casos son ideales para realizar una valoración integral, pues por su acelerada transformación y contrastes de variables urbanas y ambientales evidencian a baja escala la dinámica de la localidad, lo que se puede extrapolar al resto de la ciudad.

El objetivo de la presente investigación fue la valoración de la calidad del paisaje urbano-ambiental de Juriquilla y Santa Rosa Jáuregui en la ciudad de Querétaro, localidades en las que se realizaron videograbaciones con el fin de justipreciar indicadores urbanos y ambientales, los cuales se transformaron en capas con formato raster, producto de la interpolación de distancia inversa en un SIG. Posteriormente, se aplicó una evaluación multicriterio para obtener un mapa de calidad del paisaje ambiental y uno de calidad del paisaje urbano; dichos mapas se sumaron y se elaboró otro de la calidad del paisaje urbano-ambiental. De forma complementaria 
se definieron zonas de atención urbano-ambiental, las cuales pueden ser una alternativa de análisis y gestión territorial en futuras planeaciones.

\section{1. Área de estudio}

La investigación utilizó las localidades de Juriquilla y Santa Rosa Jáuregui, localizadas en la periferia norte de la ciudad de Querétaro (mapa 1), que presentan una superficie ocupada de 1429 ha. Al año 2010, Juriquilla y Santa Rosa Jáuregui estaban integradas por 13,309 y 18,508 habitantes, respectivamente. Forman parte de la delegación administrativa Santa Rosa Jáuregui y, en el más reciente plan parcial de desarrollo urbano 2008, ambas localidades estaban bordeadas de suelos de preservación ecológica (Gobierno Municipal de Querétaro, 2008). Actualmente presentan una intensa ocupación y cambios en el uso del suelo en la que sobresalen zonas habitacionales; en Santa Rosa Jáuregui destaca la autoconstrucción y en Juriquilla los desarrollos inmobiliarios de viviendas en serie (Hernández-Guerrero, 2015; García, 2016).

\section{Mapa 1 \\ Localización de Juriquilla y Santa Rosa Jáuregui}

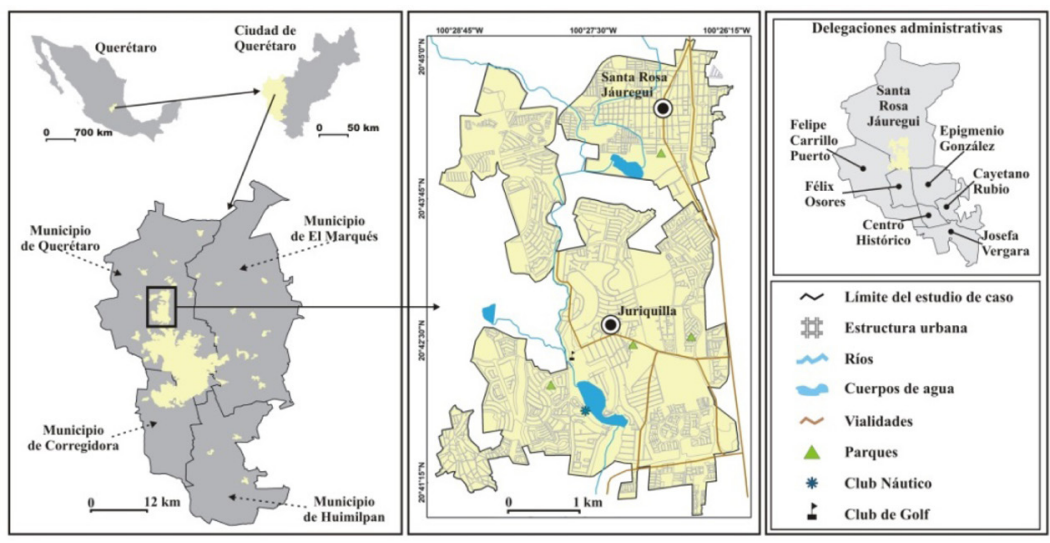

Fuente: elaboración propia con base en información del Inegi (2015b) y el software ArcGis 10.0 (ESRI, 2013).

Ambos casos de estudio comparten las siguientes características biofísicas: a) clima seco y semi-seco, con temperatura media anual de $21^{\circ} \mathrm{C}$ y precipitación anual de $520 \mathrm{~mm}$; b) la vegetación predominante es matorral xerófito y crasicaule, aunque se distinguen especies exóticas intraurbanas (jacaranda, pirul, laurel y eucalipto) y se tiene presencia de bosque tropical caducifolio en secciones contiguas; c) el tipo de suelo que 
predomina es el vertisol, suelos negros de arcillas expandibles, y $d$ ) existe un río canalizado y visiblemente contaminado que atraviesa los dos casos. En su recorrido cuenta con las presas reguladoras Dolores (Santa Rosa Jáuregui) y El Cajón (Juriquilla), la primera forma parte de un parque y la segunda se adaptó como club náutico (Hernández-Guerrero, 2015).

\section{Métodos y herramientas}

\subsection{Indicadores y criterios de valoración}

La definición de indicadores fue precedida de la división del paisaje urbano y del paisaje ambiental. Así, para determinar la calidad del paisaje urbano se definieron tres categorías y 12 indicadores, con base en los aportes de Briceño y Gil (2003), Briceño et al. (2011) y Keshtkaran et al. (2017). Por su parte, para la calidad del paisaje ambiental se adaptaron cuatro categorías y 11 indicadores de los trabajos de Pascual González y Peña Díaz (2012) y Hernández-Guerrero y Osorno (2018). En suma, son siete categorías y 23 indicadores (cuadro 1 y cuadro 2 ).

El siguiente paso fue la definición de criterios y rangos de valoración de los indicadores. En este caso, se realizaron recorridos en cuatro lugares contrastantes de los estudios de caso para registrar las particularidades de cada indicador y comprobar, corregir o adaptar el criterio de valoración. Esa actividad permitió definir cinco criterios para valorar cada indicador y cinco rangos de calidad, donde uno es muy baja calidad y se define por la presencia de uno o ningún criterio, mientras que cinco es muy alta calidad y se define con la presencia de cinco criterios (anexo I y anexo II). La valoración para la calidad del paisaje urbano se acompaña de igualdad, proximidad, regularidad, simplicidad, simetría, legibilidad y estructura (Briceño y Gil, 2003). Por su parte, la valoración para la calidad del paisaje ambiental se compone de color, escala, dimensión, contraste visual y extensión de la escena (Hernández-Guerrero, 2015).

\subsection{Recolección de datos}

El proceso se adaptó del método de Karmanov y Hamel (2008), el cual consiste en recorridos acompañados con videograbaciones para agilizar la valoración de los indicadores y contar con una mayor escena. Se utilizaron videocámaras GoPro modelo Hero 7 que, por su tamaño, anti-shock y nitidez, permitieron realizar recorridos rápidos y reducir tiempos, aunque para los lugares inseguros se utilizó la aplicación digital Street View de Google Earth Pro. Por su parte, los indicadores, criterios y rangos de valoración 


\section{Cuadro 1}

\section{Categorías, indicadores y descriptivos para la valoración de la calidad del paisaje urbano}

\begin{tabular}{|c|c|c|}
\hline Categoría & Indicador & Descriptivo \\
\hline \multirow{4}{*}{$\begin{array}{l}\text { Manzana } \\
\text { urbana }\end{array}$} & Alineamiento de fachadas & $\begin{array}{l}\text { Alineación de las fachadas de la manzana, } \\
\text { incluye continuidad del plano sin interrupcio- } \\
\text { nes por objetos salientes o volumétricos. }\end{array}$ \\
\hline & Calidad de las fachadas & $\begin{array}{l}\text { Tratamiento de las fachadas por los materiales } \\
\text { de construcción, acabados, estructura y com- } \\
\text { posición. }\end{array}$ \\
\hline & $\begin{array}{l}\text { Cantidad de mobiliario } \\
\text { urbano }\end{array}$ & $\begin{array}{l}\text { Cantidad y tipo de mobiliario urbano básico } \\
\text { como luminarias, bancas, jardineras, señalética } \\
\text { o cestos de basura. }\end{array}$ \\
\hline & $\begin{array}{l}\text { Infraestructura de servicios } \\
\text { urbanos }\end{array}$ & $\begin{array}{l}\text { Infraestructura disponible y segura para la } \\
\text { prestación de servicios básicos urbanos. }\end{array}$ \\
\hline \multirow{4}{*}{ Calles } & Funcionalidad de las calles & $\begin{array}{l}\text { Condiciones de la red viaria que facilita el } \\
\text { tránsito y movilidad. }\end{array}$ \\
\hline & Continuidad de la calle & $\begin{array}{l}\text { Acompaña la función de la red viaria a través } \\
\text { del recubrimiento uniforme, sin obstáculos, } \\
\text { accesible y con dimensiones asequibles. }\end{array}$ \\
\hline & Mantenimiento de calles & $\begin{array}{l}\text { Acciones dirigidas a preservar, conservar y } \\
\text { restaurar las calles. }\end{array}$ \\
\hline & Calidad de las aceras & $\begin{array}{l}\text { Franja entre la calle y las fachadas de condicio- } \\
\text { nes ideales para el tránsito peatonal: continuo, } \\
\text { seguro e incluyente. }\end{array}$ \\
\hline \multirow{4}{*}{$\begin{array}{l}\text { Espacios } \\
\text { abiertos }\end{array}$} & $\begin{array}{l}\text { Condiciones de accesibili- } \\
\text { dad }\end{array}$ & $\begin{array}{l}\text { Acceso público con conectividad entre personas } \\
\text { y con la red viaria, además tiene condiciones } \\
\text { incluyentes y transitables. }\end{array}$ \\
\hline & $\begin{array}{l}\text { Calidad de acabados y } \\
\text { materiales }\end{array}$ & $\begin{array}{l}\text { Condiciones de durabilidad de la estructura e } \\
\text { infraestructura y acabados óptimos en pintura } \\
\text { y herrería. }\end{array}$ \\
\hline & $\begin{array}{l}\text { Confort en equipamiento y } \\
\text { mobiliario }\end{array}$ & $\begin{array}{l}\text { El espacio dispone de bancas, cestos de basura, } \\
\text { luminarias y señaléticas, áreas verdes, equipa- } \\
\text { miento recreativo o deportivo. }\end{array}$ \\
\hline & Mantenimiento del espacio & $\begin{array}{l}\text { Acciones dirigidas a preservar, conservar y } \\
\text { restaurar el estado de los espacios abiertos. }\end{array}$ \\
\hline
\end{tabular}

Fuente: elaboración propia con base en Briceño y Gil (2003) y Hernández-Guerrero y Osorno (2018). 


\section{Cuadro 2}

\section{Categorías, indicadores y descriptivos para la valoración de la calidad del paisaje ambiental}

\begin{tabular}{|c|c|c|}
\hline Categoria & Indicador & Descriptivo \\
\hline \multirow{4}{*}{ Contaminación } & $\begin{array}{l}\text { Agua visiblemente } \\
\text { contaminada }\end{array}$ & $\begin{array}{l}\text { Ríos, arroyos, canales y cuerpos de agua en } \\
\text { el espacio público con visible contamina- } \\
\text { ción (olor y residuos sólidos). }\end{array}$ \\
\hline & Presencia de basura & $\begin{array}{l}\text { Basura presente en calles, banquetas y } \\
\text { camellones. }\end{array}$ \\
\hline & Frecuencia vehicular & $\begin{array}{l}\text { Afluencia de vehículos, camiones y moto- } \\
\text { cicletas. }\end{array}$ \\
\hline & $\begin{array}{l}\text { Presencia de } \\
\text { contaminantes visuales }\end{array}$ & $\begin{array}{l}\text { Carteles, grafiti, publicidad e infraestruc- } \\
\text { tura de servicios urbanos con deficiente } \\
\text { disposición. }\end{array}$ \\
\hline \multirow{2}{*}{$\begin{array}{l}\text { Vegetación } \\
\text { arbórea }\end{array}$} & Cantidad de árboles & $\begin{array}{l}\text { Cantidad de vegetación arbórea presente en } \\
\text { calles, banquetas, camellones y espacios } \\
\text { abiertos. }\end{array}$ \\
\hline & $\begin{array}{l}\text { Cantidad de árboles } \\
\text { dańados }\end{array}$ & $\begin{array}{l}\text { Cantidad de vegetación arbórea dañada } \\
\text { presente en calles, banquetas, camellones y } \\
\text { espacios abiertos. }\end{array}$ \\
\hline \multirow[b]{2}{*}{ Socio-culturales } & $\begin{array}{l}\text { Cantidad de sitios con } \\
\text { desperdicio de agua }\end{array}$ & $\begin{array}{l}\text { Cantidad de sitios con desperdicio de agua } \\
\text { en el espacio público. }\end{array}$ \\
\hline & $\begin{array}{l}\text { Depósitos de basura } \\
\text { clandestinos }\end{array}$ & $\begin{array}{l}\text { Depósitos no autorizados que suelen ser } \\
\text { utilizados para disposición y acumulación } \\
\text { de basura. }\end{array}$ \\
\hline \multirow{3}{*}{ Atmosférico } & Confort térmico & $\begin{array}{l}\text { Nivel de temperatura para el desarrollo de } \\
\text { actividades humanas y biológicas. Medida } \\
\text { en grados Celsius. }\end{array}$ \\
\hline & Confort acústico & $\begin{array}{l}\text { Nivel de ruido ocasionado por las activida- } \\
\text { des humanas que interviene en la salud de } \\
\text { las personas. Medida en decibelios. }\end{array}$ \\
\hline & Velocidad del viento & $\begin{array}{l}\text { Dispersa contaminantes y regula tempera- } \\
\text { tura. Se utilizó una conversión de la escala } \\
\text { de Beaufort. Medida en } \mathrm{km} / \mathrm{h} \text {. }\end{array}$ \\
\hline
\end{tabular}
(2018).

Fuente: elaboración propia con base en Briceńo y Gil (2003) y Hernández-Guerrero y Osorno

se incluyeron en una ficha de observación (anexo III) y ésta, a su vez, en tabletas digitales para agilizar el registro.

De forma complementaria se definieron 84 polígonos de referencia definidos de forma arbitraria respecto al trazado viario para realizar los recorridos de las videograbaciones. No se utilizaron zonificaciones 
preestablecidas, ya que el fin es que los propios indicadores generen las zonificaciones finales través de los niveles de calidad del paisaje.

Las videograbaciones se realizaron con recorridos en forma de espiral por cada polígono de referencia para tener la totalidad de la superficie. Cabe señalar que casi la totalidad de los registros de las valoraciones se realizaron con las videograbaciones, excepto cuatro indicadores que se registraron in situ en cuatro puntos de cada polígono, es el caso de frecuencia vehicular, confort térmico, confort acústico y velocidad del viento.

La valoración se llevó a cabo por un mínimo de tres evaluadores para tener un registro impar y minimizar el sesgo en el resultado (Stewart et al., 1983); los evaluadores fueron ajenos a los estudios de caso (con experiencia en el tema) y los resultados se promediaron. Las videograbaciones y la valoración in situ se realizaron en recorridos matutinos (8:00-11:00 h) y vespertinos (14:00-17:00 h): seis videograbaciones y seis registros de indicadores in situ. El levantamiento de información fue de 14 polígonos por semana (seis semanas), ello arrojó 504 fichas para los 84 polígonos. No obstante, se realizó una segunda valoración en 18 polígonos con el fin de corroborar algunos registros inciertos. En total se obtuvieron 612 fichas de observación con los registros de valoración para cada indicador.

\subsection{Procesamiento e interpolación de datos}

Con base en Buzai (2011), los registros de valoración se definieron a través del centroide de cada polígono para determinar la influencia de la calidad del paisaje (mapa 2). Adicional a ello se elaboraron las bases de datos por indicadores con su respectiva división entre calidad del paisaje urbano y calidad del paisaje ambiental. Esas bases de datos se manipularon en un Sistema de Información Geográfica (SIG) (ArcMap 10) para ser transformadas a datos de tipo vector y generar capas (layers) de puntos de cada indicador.

La siguiente etapa corresponde a la interpolación por indicador; debido a la naturaleza de los datos obtenidos (no geoestadísticos) se optó por utilizar una técnica determinística a través de interpolación de distancia inversa (IDW, por sus siglas en inglés inverse distance weighted) (Fuenzalida, 2015). Al ser una técnica de interpolación determinística y establecida como interpolador exacto, es posible la construcción de mallas de puntos con valores asociados a la extensión de similitudes, donde los puntos próximos a la muestra son más parecidos entre sí, de tal forma que existe una relación lineal de similitud entre un punto de valor conocido y sus vecinos (Castro et al., 2018). Esta técnica generó la continuidad espacial de 84 puntos por indicador, con un rango de celda de $10 \times 10 \mathrm{~m}$, debido a que 


\section{Mapa 2}

Estudios de caso con los centroides de los registros de la valoración

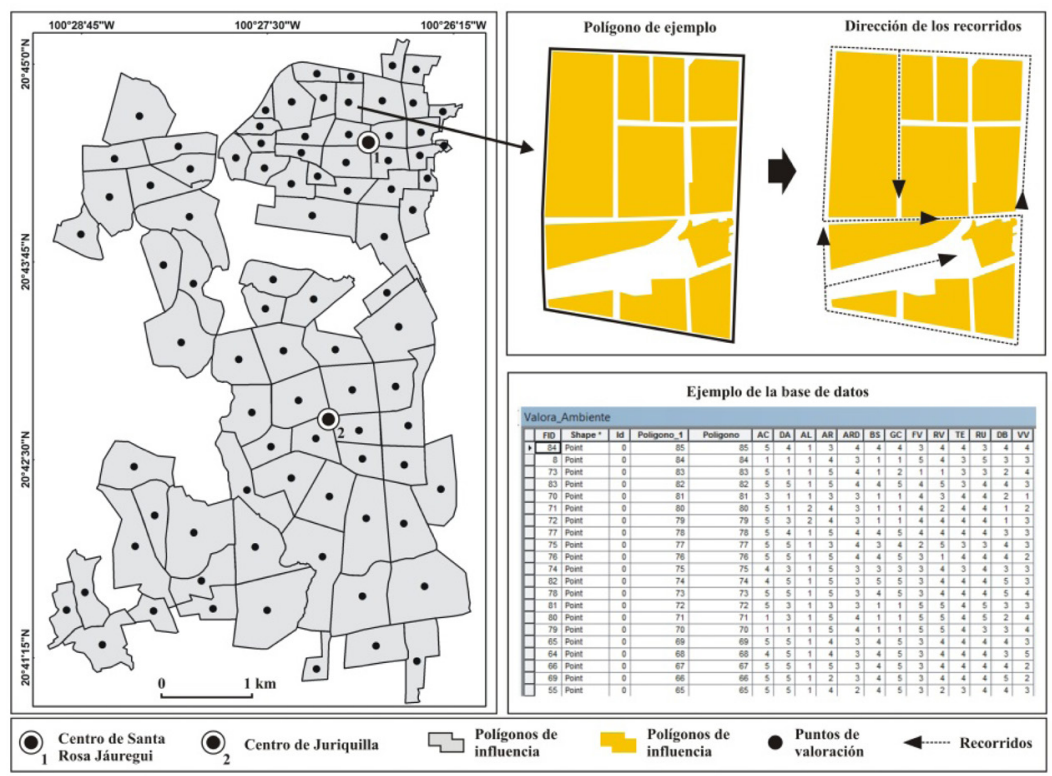

Fuente: elaboración propia con base en datos del Inegi (2015b) y el software ArcGis 10.0 (ESRI, 2013).

el rango de proximidad se reduciría entre valoraciones de cada punto dentro de la capa (ecuación 1).

$$
\hat{z}\left(x_{j}\right)=\frac{\sum_{i=1}^{n} z\left(x_{i}\right) \cdot d_{i j}^{-\alpha}}{\sum_{i=1}^{n} d_{i j}^{-\alpha}}
$$

Donde, $\hat{z}$ es el valor estimado en el punto, $x_{j}$ es el punto en lo que se estima el valor del indicador, $x_{i}$ son los puntos muestrales vecinos, $z$ es el valor observado del indicador en el punto muestral dentro de la región de vecindad, $n$ es el número de puntos muestrales utilizados en la estimación, $d_{i j}$ es la distancia euclidiana entre $x_{i}$ y $x_{i}$ y $\alpha$ es el exponente de ponderación (se utilizó $\alpha=2$ ).

El resultado de esta fase fue una capa en formato raster por cada indicador; cada una de ellas fue reclasificada para seguir manteniendo el rango de 1 a 5 . Este proceso es necesario, pues la reclasificación cataloga o modifica los valores del raster antiguo por valores alternativos y siempre se aplica a toda la celda dentro de una zona (Buzai, 2015; Varatharajan et al., 2018), ello permitió mantener los valores deseados dentro del intervalo. 


\subsection{Evaluación multicriterio}

La evaluación multicriterio es un método de superposición cartográfica ponderada de capas raster para seleccionar sitios y priorizar zonas o modelos de adecuación; el proceso de evaluación implica la realización de un modelo para resolver un problema, así como su división en submodelos, la identificación de las capas de entrada (capas con rango en común, de lo contrario serán reclasificadas), la ponderación entre indicadores y la definición del peso por capa (indicador); de esta manera, cada capa se multiplica por el peso y después se suman para obtener un solo resultado para ser validado (Hanafi-Bojd et al., 2012; Buzai, 2015; Varatharajan et al., 2018).

Luego de lo anterior, el siguiente paso fue la definición del peso para cada uno de los indicadores de entrada; esta actividad se realizó mediante un análisis de comparación pareada con ayuda del programa Super Decisions (Creative Decisions Foundation, 2019) [software de acceso libre que utiliza el Proceso de Análisis Jerárquico (AHP por sus siglas en inglés, analytic hierarchy process)], en el que se establecieron ponderaciones de los indicadores para definir su influencia respecto a otro y determinar el peso del indicador.

Con base en Buzai (2015), la obtención de los pesos se acompañó del conocimiento en materia urbana y ambiental del equipo de trabajo del presente estudio (geografía ambiental, ecología urbana y ordenamiento territorial), así como de la experiencia in situ y la literatura consultada; se realizaron combinaciones y se definieron los pesos de los indicadores para la calidad del paisaje urbano y ambiental (cuadros 3 y 4).

Una vez que se obtuvieron los pesos, la siguiente etapa fue la implementación de una suma lineal ponderada con los indicadores del paisaje urbano y otra para los indicadores del paisaje ambiental, donde se superpusieron las capas raster (indicadores) y se multiplicaron por el peso antes establecido (Buzai, 2015; Kaliraj et al., 2015), este ejercicio se realizó con el módulo raster calculator del programa ArcGis 10 (ESRI, 2013), donde se incluyeron las ecuaciones 2 y 3 que, a través de álgebra de mapas, generaron los raster de salida, esto es, la definición de los mapas de salida para la calidad del paisaje urbano y para la calidad del paisaje ambiental. 


\section{Cuadro 3}

Definición de los pesos para los indicadores del paisaje urbano

\begin{tabular}{|c|l|c|c|c|}
\hline \multicolumn{1}{|c|}{ Categoría } & \multicolumn{1}{|c|}{ Indicador } & Clave & Peso & $\%$ \\
\hline \multirow{5}{*}{ Manzanas urbanas } & Alineamiento de fachadas & AF & 0.19954 & 19.954 \\
\cline { 2 - 6 } & Calidad de las fachadas & CF & 0.14040 & 14.04 \\
\cline { 2 - 6 } & $\begin{array}{l}\text { Cantidad de mobiliario } \\
\text { urbano }\end{array}$ & MU & 0.11138 & 11.138 \\
\cline { 2 - 6 } & $\begin{array}{l}\text { Infraestructura de servicios } \\
\text { urbanos }\end{array}$ & IM & 0.13528 & 13.528 \\
\hline \multirow{5}{*}{ Calles } & \begin{tabular}{l} 
Funcionalidad de las calles \\
\cline { 2 - 6 }
\end{tabular} & FC & 0.05051 & 5.051 \\
\cline { 2 - 6 } & Continuidad de la calle & CC & 0.04308 & 4.308 \\
\cline { 2 - 6 } & Mantenimiento de la calle & MC & 0.05169 & 5.169 \\
\cline { 2 - 6 } & Calidad de las aceras & AC & 0.08060 & 8.06 \\
\hline & $\begin{array}{l}\text { Condiciones de accesibili- } \\
\text { dad }\end{array}$ & AE & 0.05709 & 5.709 \\
\cline { 2 - 6 } & $\begin{array}{l}\text { Calidad de acabados y mate- } \\
\text { riales }\end{array}$ & AM & 0.04357 & 4.357 \\
\cline { 2 - 6 } & $\begin{array}{l}\text { Confort en equipamiento y } \\
\text { mobiliario }\end{array}$ & EM & 0.05048 & 5.048 \\
\hline & Mantenimiento del espacio & ME & 0.03638 & 3.638 \\
\hline
\end{tabular}

Fuente: elaboración propia con base en los registros de la calidad del paisaje urbano.

\section{Cuadro 4}

Definición de los pesos para los indicadores del paisaje ambiental

\begin{tabular}{|c|c|c|c|c|}
\hline Categoria & Indicador & Clave & Peso & $\%$ \\
\hline \multirow{4}{*}{ Contaminación } & $\begin{array}{l}\text { Agua visiblemente } \\
\text { contaminada }\end{array}$ & $\mathrm{AC}$ & 0.07274 & 7.274 \\
\hline & $\begin{array}{l}\text { Presencia de basura en las } \\
\text { calles }\end{array}$ & $\mathrm{BC}$ & 0.13979 & 13.979 \\
\hline & Frecuencia vehicular & $\mathrm{FV}$ & 0.11629 & 11.629 \\
\hline & $\begin{array}{l}\text { Presencia de contaminantes } \\
\text { visuales }\end{array}$ & $\mathrm{CV}$ & 0.04392 & 4.392 \\
\hline \multirow{2}{*}{$\begin{array}{l}\text { Vegetación } \\
\text { arbórea }\end{array}$} & Cantidad de árboles & AR & 0.20797 & 20.797 \\
\hline & $\begin{array}{l}\text { Cantidad de árboles } \\
\text { dañados }\end{array}$ & ARD & 0.12297 & 12.297 \\
\hline
\end{tabular}


Cuadro 4 (continuación)

\begin{tabular}{|c|l|l|c|c|}
\hline Categoría & \multicolumn{1}{|c|}{ Indicador } & \multicolumn{1}{c|}{ Clave } & \multicolumn{1}{c|}{ Peso } & $\%$ \\
\hline \multirow{3}{*}{ Socio-cultural } & $\begin{array}{l}\text { Cantidad de sitios con } \\
\text { desperdicio de agua }\end{array}$ & DA & 0.04731 & 4.731 \\
\cline { 2 - 5 } & $\begin{array}{l}\text { Depósitos de basura } \\
\text { clandestinos }\end{array}$ & DB & 0.07323 & 7.323 \\
\hline \multirow{3}{*}{ Atmosférico } & Confort térmico & CT & 0.05639 & 5.639 \\
\cline { 2 - 5 } & Confort acústico & CA & 0.07541 & 7.541 \\
\cline { 2 - 5 } & Velocidad del viento & VV & 0.04398 & 4.398 \\
\hline & & Total & 1 & 100 \\
\hline
\end{tabular}

Fuente: elaboración propia con base en los registros de la calidad del paisaje ambiental.

$$
\begin{aligned}
& C P U=\left[\left(A F_{c} * A F_{f}\right)+\left(C F_{c} * C F_{f}\right)+\left(M U_{c} * M U_{f}\right)+\left(I M_{c} * I M_{f}\right)+\left(F C_{c}\right.\right. \\
& \left.* F C_{f}\right)+\left(C C_{c} * C C_{f}\right)+\left(M C_{c} * M C_{f}\right)+\left(A C_{c} * A C_{f}\right)+\left(A E_{c} * A E_{f}\right)+\left(A M_{c} *\right. \\
& \left.\left.A M_{f}\right)+\left(E M_{c} * E M_{f}\right)+\left(M E_{c} * M E_{f}\right)\right]
\end{aligned}
$$

Donde $C P U$ es calidad de paisaje urbano, $A F$ es alineamiento de fachadas, $C F$ es calidad de fachadas, $M U$ es mobiliario urbano, $I M$ es infraestructura en la manzana, $F C$ es funcionalidad de la calle, $C C$ es continuidad de la calle, $M C$ es mantenimiento de la calle, $A C$ es calidad de acera, $A E$ es accesibilidad del espacio abierto, $A M$ es acabados y materiales, $E M$ es equipamiento y mobiliario y $M E$ es mantenimiento del espacio abierto. El sufijo con la letra $c$ indica el peso de escala preestablecido, según las características de la capa, mientras que el sufijo con la letra $f$ indica el porcentaje de peso de influencia resultante de la ponderación pareada.

$$
\begin{aligned}
& C P A=\left[\left(A C_{c} * A C_{f}\right)+\left(B C_{c} * B C_{f}\right)+\left(F V_{c} * F V_{f}\right)+\left(C V c * C V_{f}\right)+\left(A R_{c} * A R_{f}\right.\right. \\
& )+\left(A R D_{c} * A R D_{f}\right)+\left(D A_{c} * D A_{f}\right)+\left(D B_{c} * D B_{f}\right)+\left(C T_{c} * C T_{f}\right)+\left(C A_{c} *(3)\right. \\
& \left.\left.C A_{f}\right)+\left(V V_{c} * V V_{f}\right)\right]
\end{aligned}
$$

Donde $C P A$ es calidad del paisaje ambiental, $A C$ es agua visiblemente contaminada, $B C$ es basura en la calle, $F V$ es frecuencia vehicular, $C V$ es contaminantes visuales, $A R$ es árboles, $A R D$ es árboles dañados, $D A$ es desperdicio de agua, $D B$ es depósitos de basura clandestinos, $C T$ es confort térmico, $C A$ es confort acústico y $V V$ es velocidad del viento. El sufijo con la letra $c$ indica el peso de escala preestablecido según las características de la capa, mientras que el sufijo con la letra $f$ indica el porcentaje de peso de influencia resultante de la ponderación pareada. 
Con base en los resultados de la aplicación de las ecuaciones anteriores se generó y diseñó un mapa de calidad del paisaje urbano y otro mapa de calidad del paisaje ambiental. Los dos se sumaron con álgebra de mapas y se reclasificaron en los cinco rangos preestablecidos para obtener el mapa final, que contiene la valoración de la calidad del paisaje urbano-ambiental.

\section{Resultados}

En la calidad del paisaje urbano, todas las categorías para Santa Rosa Jáuregui presentaron muy baja calidad, mientras que en Juriquilla predominó la calidad alta, a excepción de las categorías de calles y espacios abiertos, las cuales fueron de baja calidad. Los 12 indicadores que conforman la calidad del paisaje urbano, tanto en Juriquilla como en Santa Rosa Jáuregui, tuvieron una tendencia de calidad media, donde destacan por su condición negativa los indicadores de calles y espacios abiertos (figura 1).

De manera específica, todos los indicadores para Santa Rosa Jáuregui presentaron de calidad media a muy baja; la calidad baja y muy baja cubren $75 \%$ de la superficie, mientras que en Juriquilla cuatro indicadores presentaron calidad media y cuatro calidad baja, lo que equivale a $56 \%$ y $39 \%$ de la superficie, respectivamente.

En lo que respecta a la calidad del paisaje ambiental, Santa Rosa Jáuregui presentó calidad baja y Juriquilla calidad media. Asimismo, las categorías con menor calidad en Santa Rosa Jáuregui recaen en contaminación, vegetación arbórea y socio-cultural, mientras que en Juriquilla fueron las categorías contaminación y atmosférico (figura 2). Asimismo, en Santa Rosa Jáuregui, siete de 11 indicadores presentaron calidad baja y muy baja, los cuales corresponden a más del $75 \%$ de su superficie, mientras que en Juriquilla cuatro indicadores presentaron baja calidad, con un rango de influencia en $56 \%$ de la superficie.

De manera general, el mapa de calidad del paisaje urbano y el mapa de calidad ambiental denotaron algunos rasgos contrastantes: en Juriquilla la distribución de la calidad baja se encuentra en el centro y en la periferia, coinciden con el antiguo poblado (centro de la localidad) y asentamientos de bajos recursos económicos respectivamente, pero en el caso de Santa Rosa Jáuregui, el antiguo poblado (centro de la localidad) tiene calidad media y la periferia presenta calidad media y muy baja (figura 3, A y B). Así, en Santa Rosa Jáuregui, tanto en paisaje urbano como ambiental, predominó la calidad baja y muy baja en $93 \%$ y $88 \%$ de la superficie, respectivamente, mientras que en Juriquilla se presentaron niveles de calidad media a muy alta en $91 \%$ de la superficie; fue la calidad alta la de mayor predominio con la representación de $68 \%$ de la superficie. 


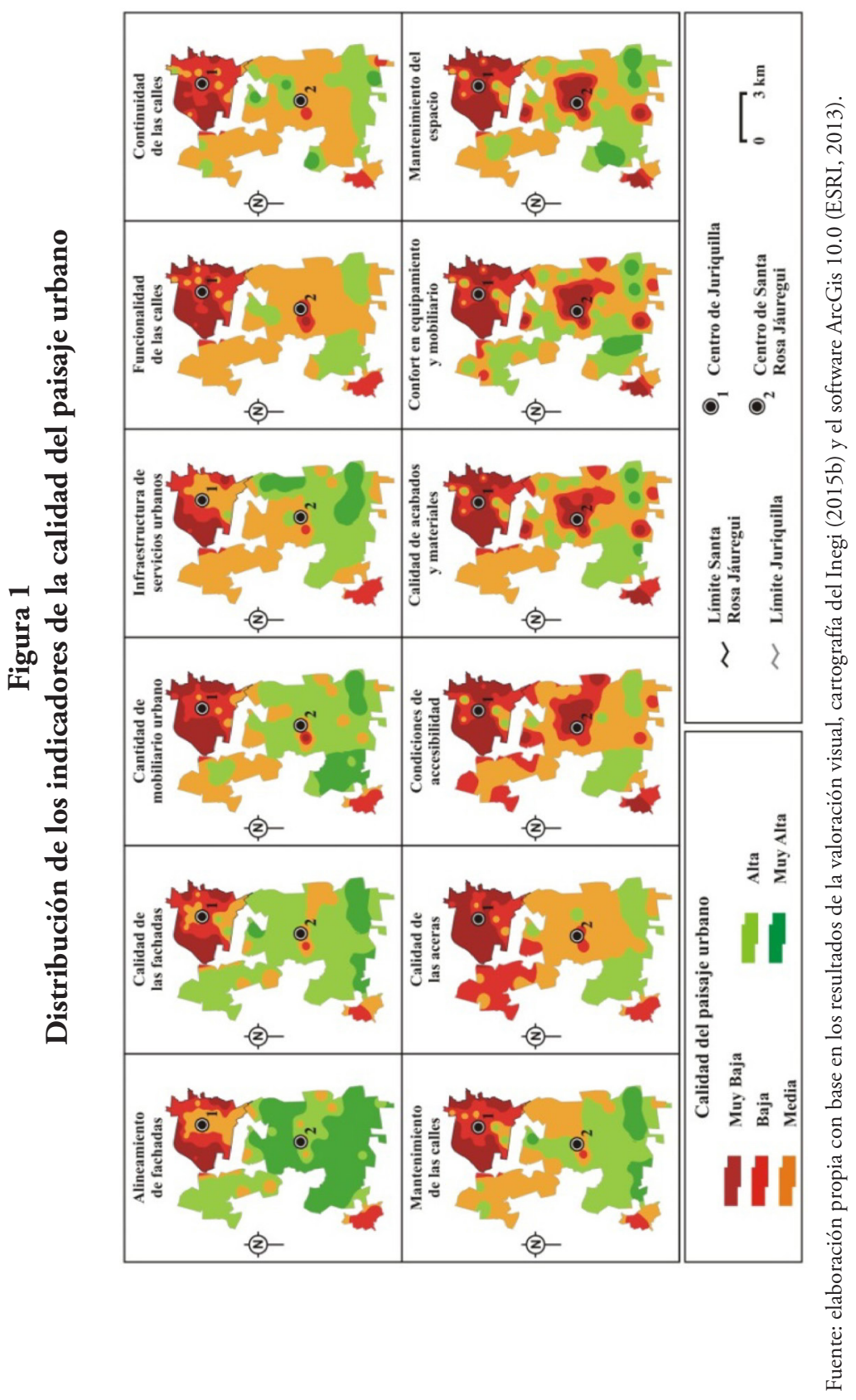




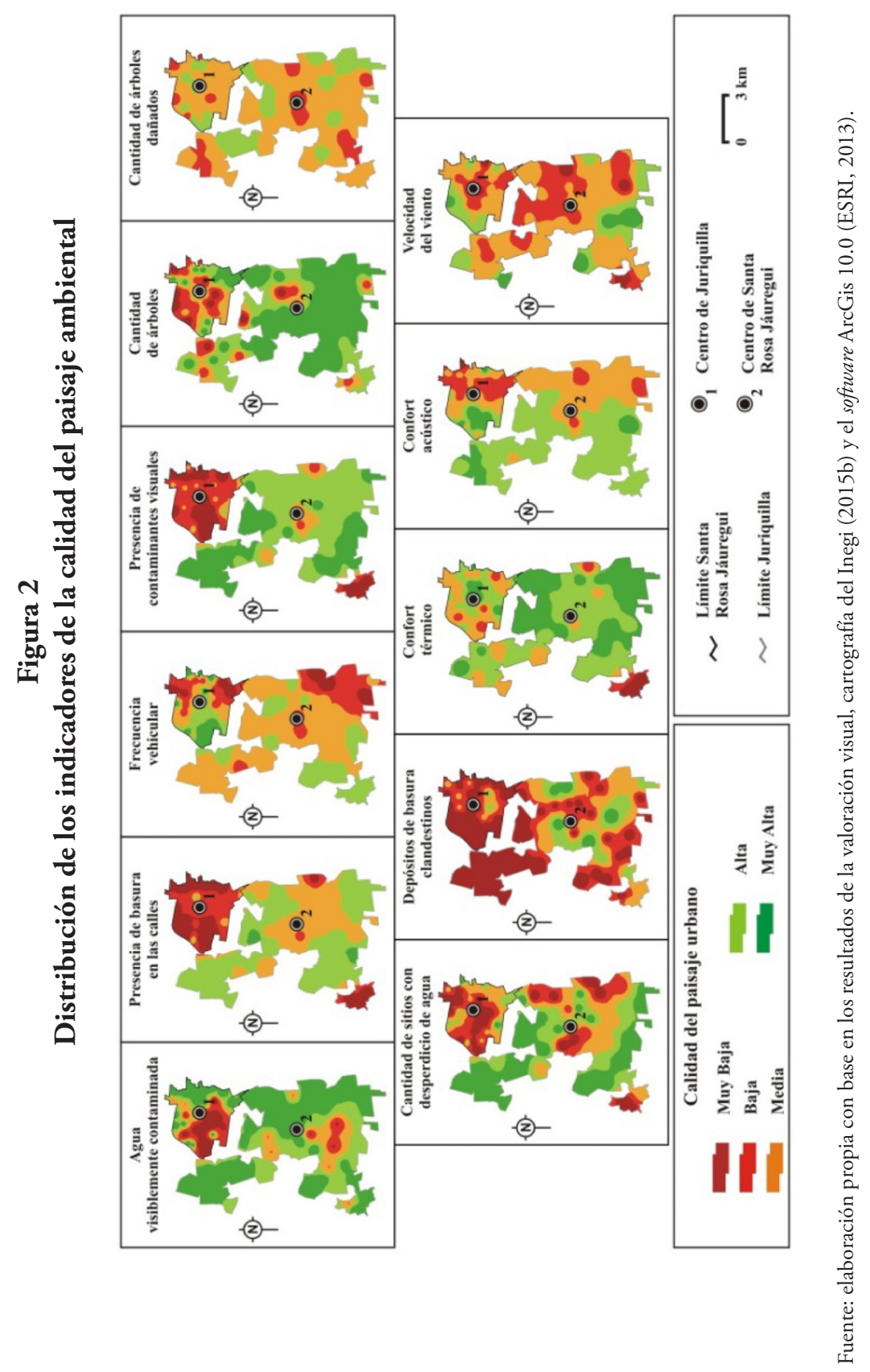




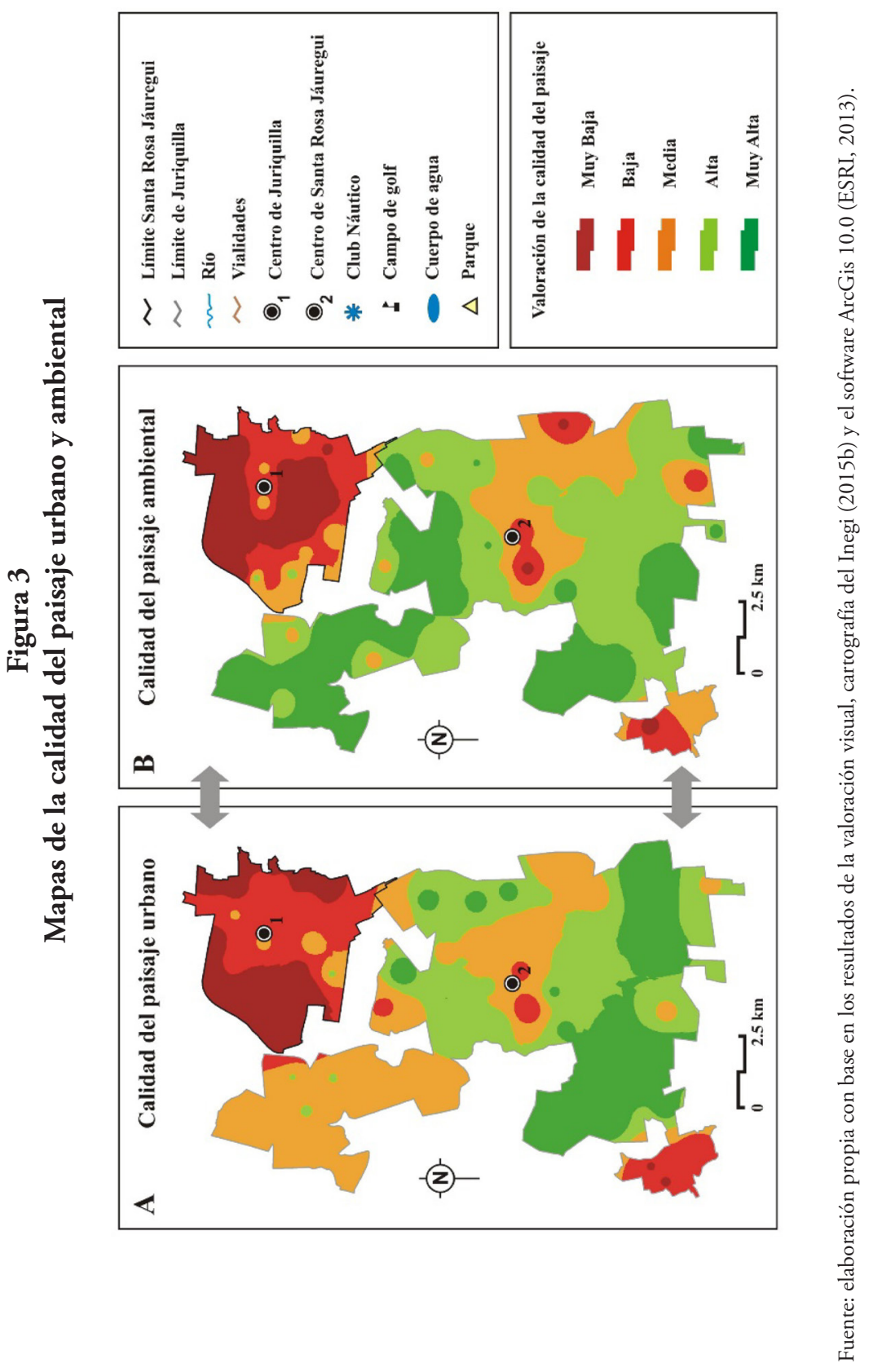


En el mapa 3, el de la calidad urbano-ambiental, se acentúan los problemas y satisfactores de los indicadores valorados. $\mathrm{Al}$ respecto, se puede señalar que las secciones con nivel de calidad bajo y muy bajo pueden considerarse zonas prioritarias para realizar acciones inmediatas que atiendan todos los indicadores valorados (mapa 3, A y B). La calidad media podría formar zonas intermedias donde las acciones de mejora sean en periodos prolongados o pausados (mapa 3, C y D). En el caso de la calidad alta y muy alta, podrían definirse zonas de monitoreo, las cuales requieren de vigilancia constante para preservar y mantener el paisaje actual (mapa 3, E y F). Así, en Santa Rosa Jáuregui, 86\% de la superficie se relaciona con zonas prioritarias, mientras que en Juriquilla sólo 11\% tendría zonas prioritarias, pues destacan las zonas de monitoreo $58 \%$ de la superficie del suroeste) y zonas intermedias (31\% de la superficie entre centro y norte).

\section{Mapa 3}

\section{Calidad del paisaje y zonas de atención urbano-ambiental}

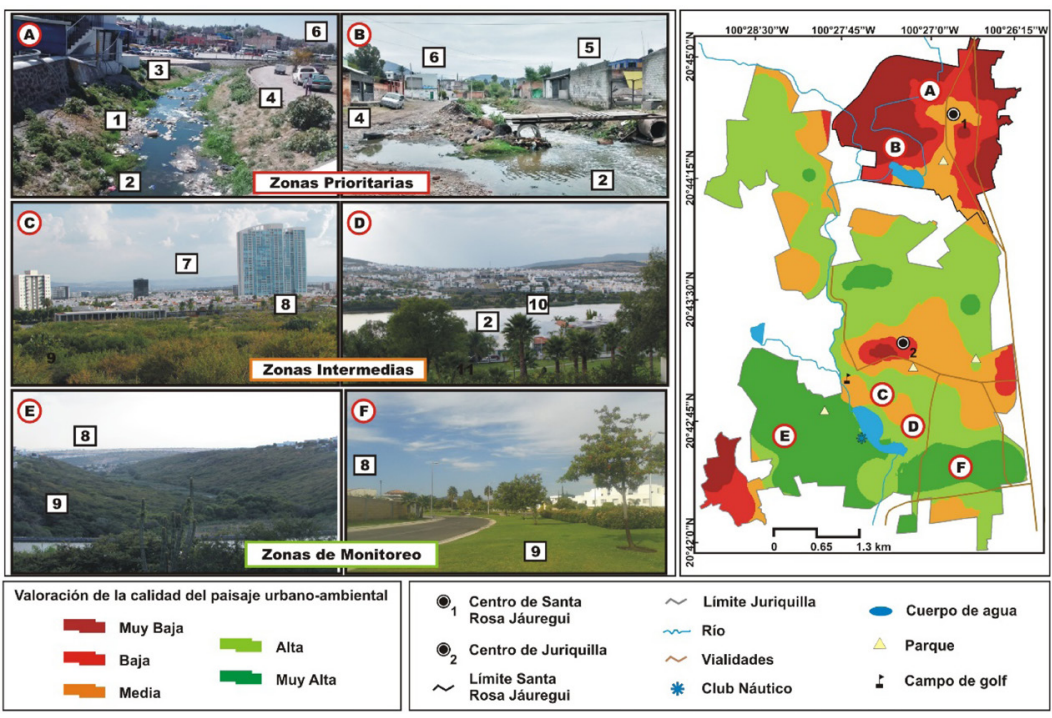

Identificadores: 1, Basura; 2, agua visiblemente contaminada; 3, fachadas deficientes; 4, vialidad poco funcional; 5 , contaminantes visuales; 6 , fachadas desalineadas; 7 , obstrucción del paisaje; 8 , vegetación arbórea; 9, espacio abierto; 10, cuerpo de agua; 11, confort térmico.

Fuente: elaboración propia con fotografías y videograbaciones del autor e información vectorial del Inegi (2015b) y el software ArcGis 10.0 (ESRI, 2013). 


\section{Discusión}

La técnica de interpolación de distancia inversa facilitó la relación de información con un mínimo de sesgo debido a la extensión de las similitudes y a los registros por cada indicador que incluyen ponderaciones a través de criterios de decisión, como lo señalan Buzai (2015) y Varatharajan et al. (2018), los registros entre vecinos se ajustaron con mayor exactitud y no excedieron el rango de valoración. Por lo mismo, los resultados tuvieron mejor definición que los establecidos por Hernández-Guerrero y Osorno (2018), ya que además de la interpolación se agregó la suma lineal ponderada con la opinión de expertos y la experiencia del trabajo in situ.

En general, el estudio presentó tres aportes que mejoraron los resultados de estudios similares: el primero fue la valoración y registro de indicadores urbanos cuyos criterios -a diferencia del aporte de Briceño y Gil (2003) y Keshtkaran et al. (2017) - se diseñaron de manera cuantitativa, y la valoración se registró de forma ágil, dinámica y con mayor rango de escena.

El segundo aporte fue la adaptación de indicadores ambientales de Hernández-Guerrero y Osorno (2018); los registros tuvieron una reducción de escala y recorridos cortos que permitieron mejorar la valoración y aproximarse a la realidad, ejemplo de ello fue que, en ese mismo trabajo, Santa Rosa Jáuregui se definió como de calidad media y en el presente trabajo fue de mala calidad, mientras que Juriquilla pasó de calidad media a buena. El tercer aporte fue el uso de videograbaciones y la herramienta de Street View de Google Earth Pro, con ello los tiempos se redujeron de manera importante, pues en el caso de Hernández-Guerrero (2015), el tiempo de valoración de indicadores fue de un año, mientras que en el presente trabajo fue de cuatro meses, con lo que se cumple la expectativa de optimizar la valoración.

Por otro lado, la determinación de zonificaciones a través de los niveles de calidad del paisaje urbano-ambiental permite argumentar detalles sobre estrategias a corto, mediano y largo plazos. En zonas prioritarias, las estrategias son a corto plazo y están dirigidas al suministro y mantenimiento de infraestructura, equipamiento, servicios y mobiliario urbano, así como a disminuir el desperdicio de agua, cuidar la vegetación arbórea y limpiar las calles. En las zonas intermedias la atención es a corto y mediano plazos, con acciones dirigidas a mejorar la funcionalidad y recubrimiento de las vialidades, construcción y mantenimiento de espacios abiertos, disminuir el desperdicio de agua, mantener la vegetación arbórea y concientizar sobre residuos sólidos. La monitorización de las zonas es 
una medida de largo plazo y se requiere en Juriquilla, como vigilancia para preservar la función de la dinámica del paisaje.

La valoración de la calidad del paisaje urbano-ambiental puede apoyar el diseño de políticas públicas y la operatividad de acciones o estrategias urbanas, así como evidenciar la omisión o ausencia de restricciones urbanas y ambientales. Ejemplo de lo anterior, entre Juriquilla y Santa Rosa Jáuregui existe una amplia zona sin construir (forma parte de una zona intermedia), y aun cuando es señalada para uso habitacional por el plan parcial de desarrollo urbano (Gobierno Municipal de Querétaro, 2008), el presente trabajo la considera como zona de amortiguamiento a largo plazo. Otro ejemplo es la identificación de baldíos subutilizados, descuidados e inseguros en zonas prioritarias, los cuales pueden ser aprovechados como jardines, control de avenidas para mitigar inundaciones, fomentar la biodiversidad y realizar actividades de recreación o educativas.

En las áreas centrales de los dos estudios de caso se localizan los antiguos poblados, que resultaron con calidad muy baja. En Santa Rosa Jáuregui se observó que el paisaje está acompañado de evidentes problemas de pobreza, segregación, desigualdad e inseguridad; por lo mismo, parece ser que la mejora de la calidad del paisaje pasa a segundo plano. Por el contrario, la calidad muy alta se encuentra en el sur de Juriquilla, acompañada de zonas habitacionales de reciente creación y circundadas por áreas verdes; en ella se observó que la calidad del paisaje urbano-ambiental por condiciones económicas, políticas y culturales -las mejoras al paisaje permiten conservar y aumentar la plusvalía- se asocia con un mercado ambiental costoso y poco incluyente.

\section{Conclusiones}

La valoración de la calidad del paisaje urbano-ambiental permitió determinar que Santa Rosa Jáuregui está representada por niveles bajos y muy bajos; prácticamente todos los indicadores urbanos y ambientales incidieron de forma negativa en el resultado, por esta razón se establecieron zonas de atención prioritaria en la mayoría de su superficie. Por su parte, en Juriquilla la calidad del paisaje urbano-ambiental se encuentra entre los niveles medio y muy alto, los cuales influyen en la determinación de zonas de atención intermedia y de monitoreo.

En lo que respecta a los métodos, la aplicación de videograbaciones, el uso de interpolación de distancia inversa y el método de evaluación multicriterio contribuyeron para que el proceso fuera ágil y los resultados detallados. El presente proceso puede ser elaborado por conocedores y población en general con previa instrucción, o bien, técnicos y especialistas 
que incluyan indicadores para robustecer el análisis en futuros estudios. Por lo tanto, el aporte metodológico puede ser replicable en otras ciudades con características similares, o bien puede ser adaptado hacia áreas más grandes manteniendo procedimientos ágiles, sencillos y con ejecución de corto tiempo.

El análisis de indicadores y la generación de mapas de la calidad del paisaje urbano-ambiental pueden apoyar instrumentos de planeación territorial, diagnóstico ambiental, políticas públicas, manejo y gestión de recursos naturales en espacios urbanos. Asimismo, la calidad del paisaje urbano-ambiental sería un complemento para analizar su funcionalidad, en continua participación entre sociedad y gobierno: 1) detección de sitios para incorporar equipos de medición ambiental, reforestaciones, mantenimiento u otros; 2) reconocimiento de las condiciones de salubridad, seguridad, estética y funcionalidad del entorno público; 3) estrategias para el diseño, reglamentos y normas de la imagen urbana; 4) mantenimiento del equilibrio y funcionalidad ecológico-urbano; 5) crecimiento urbano en armonía con el ambiente, y 6) preservación de los recursos paisajísticos y ambientales.

\section{Artículo derivado del proyecto}

El presente estudio forma parte del proyecto titulado "Nuevas geografías de la urbanización en México: Transformaciones territoriales y medios de vida de sectores sociales vulnerables en las periferias de ciudades medias", que contó con financiamiento del PAPIIT-UNAM.

\section{Agradecimientos}

El autor agradece a los evaluadores anónimos y al comité editorial de la revista, ya que gracias a sus pertinentes comentarios el manuscrito presenta mayor claridad. Asimismo, se agradece al doctor Antonio Vieyra y a la doctora Yadira Méndez Lemus por la colaboración y apoyo en el grupo de trabajo. 


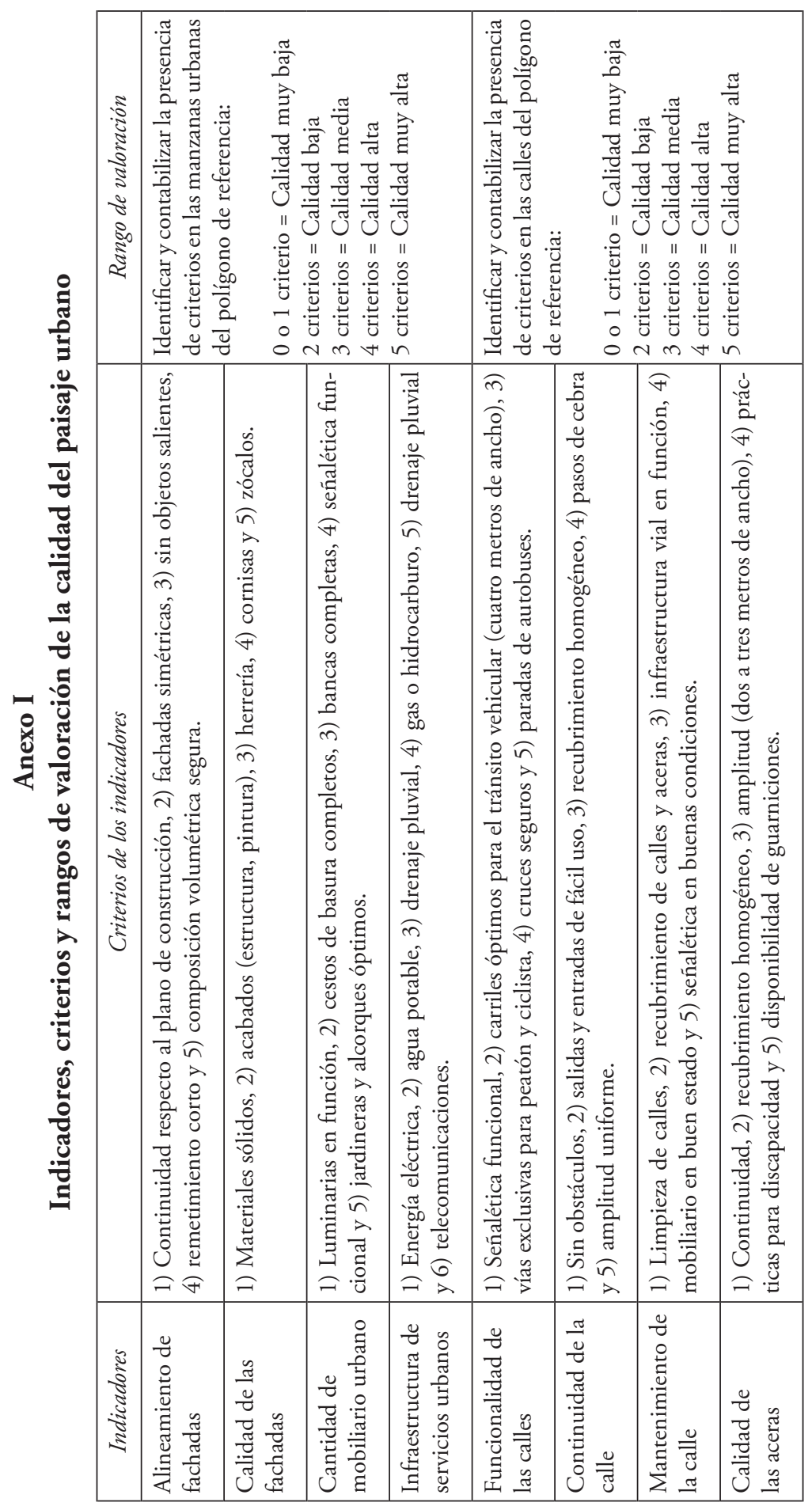




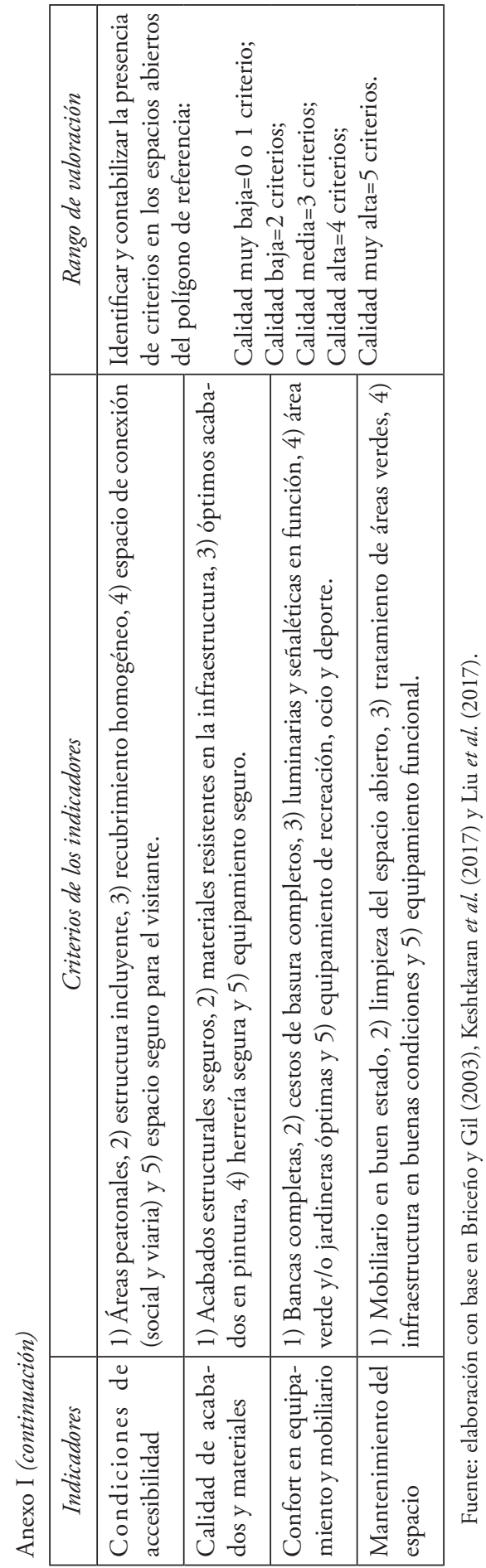




\begin{tabular}{|c|c|c|c|c|c|c|}
\hline \multirow[t]{3}{*}{ 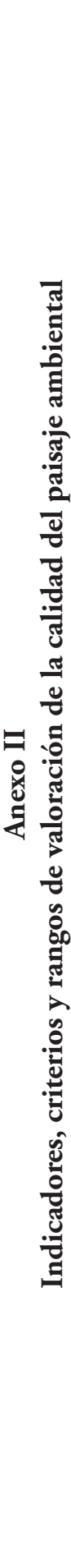 } & 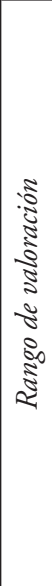 & 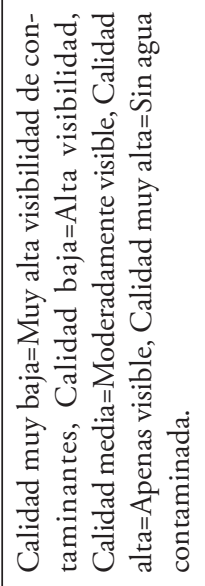 & 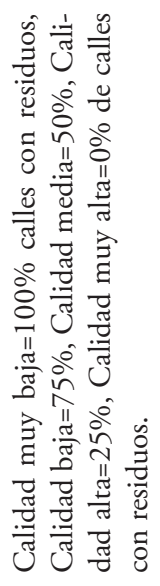 & 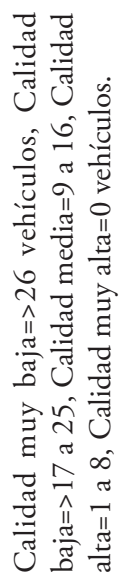 & 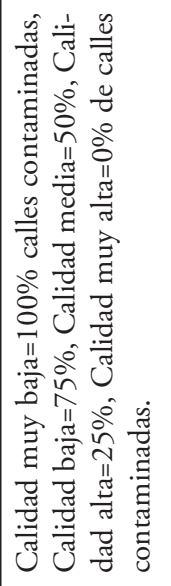 & 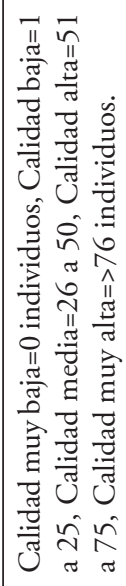 \\
\hline & 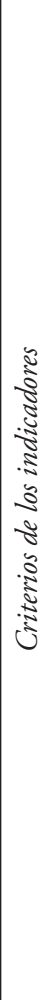 & 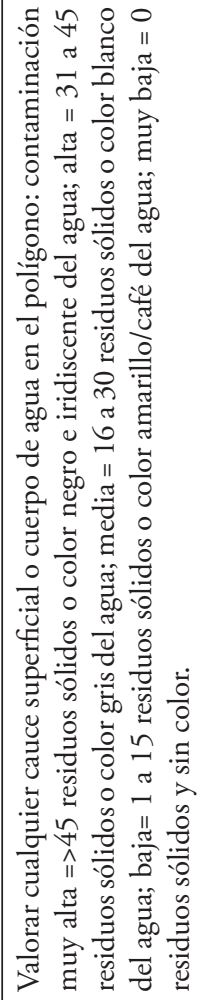 & 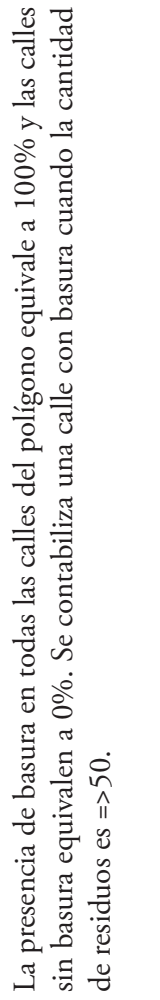 & 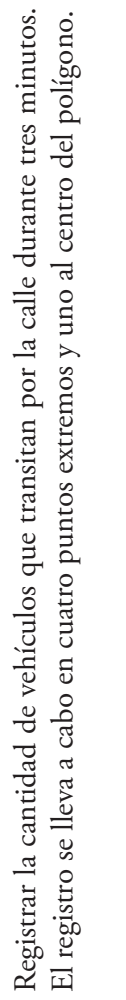 & 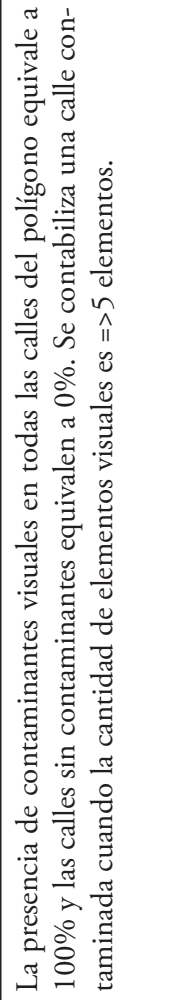 & 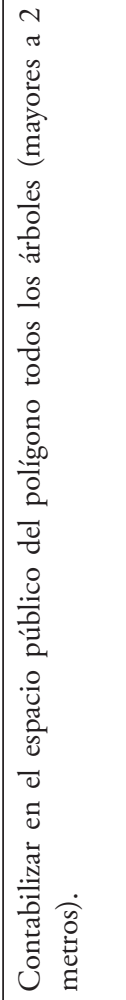 \\
\hline & 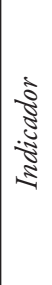 & 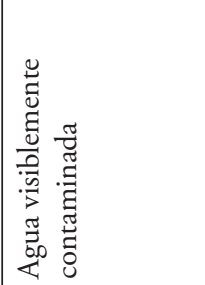 & 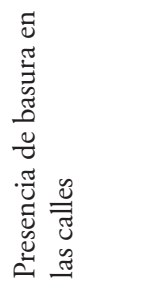 & 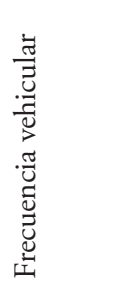 & 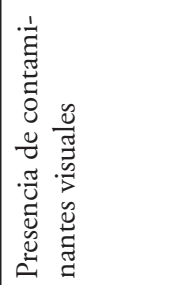 & 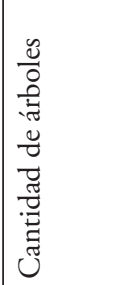 \\
\hline
\end{tabular}




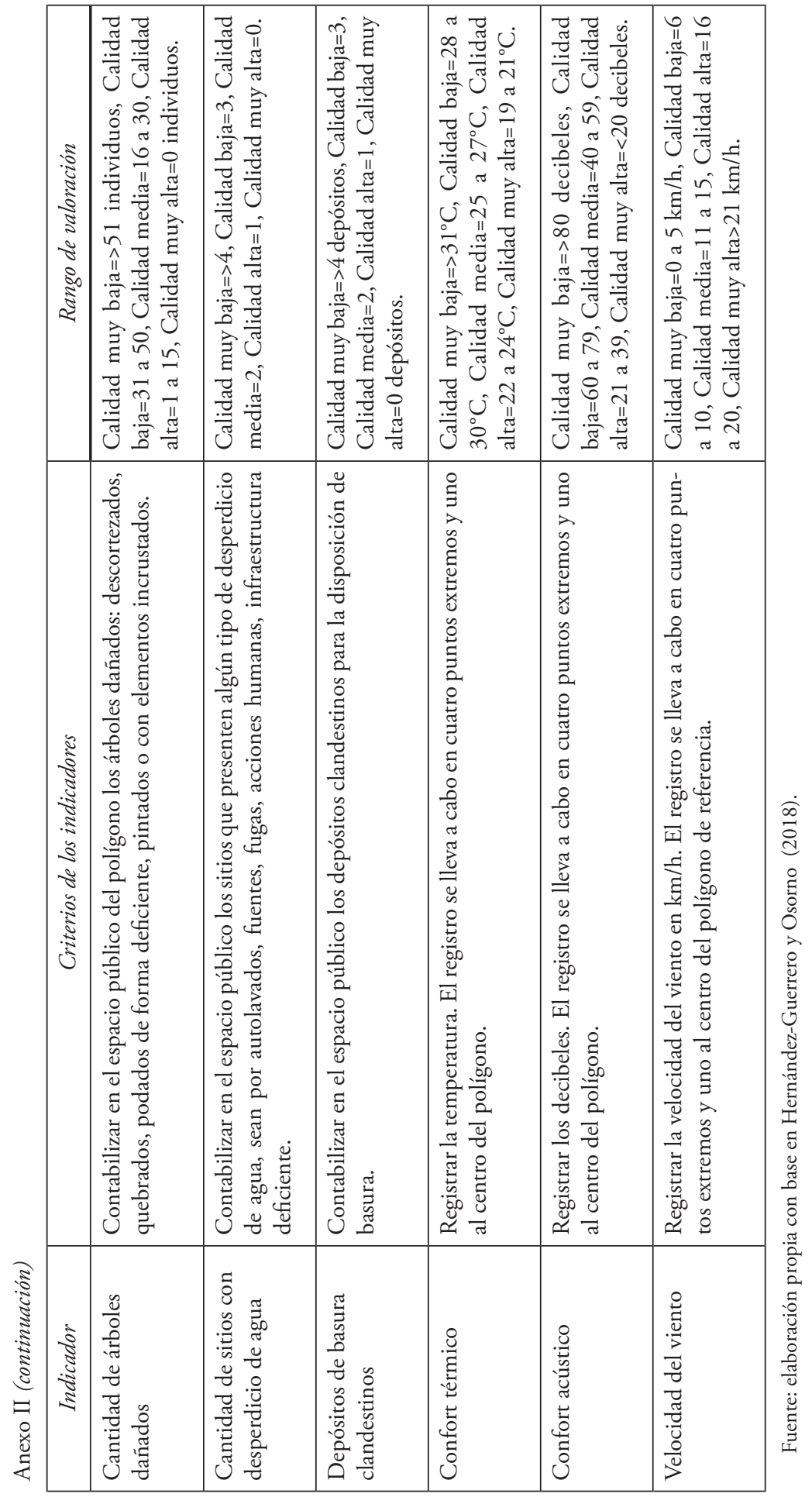




\section{Anexo III}

Ficha de observación para registrar la calidad del paisaje urbano y ambiental

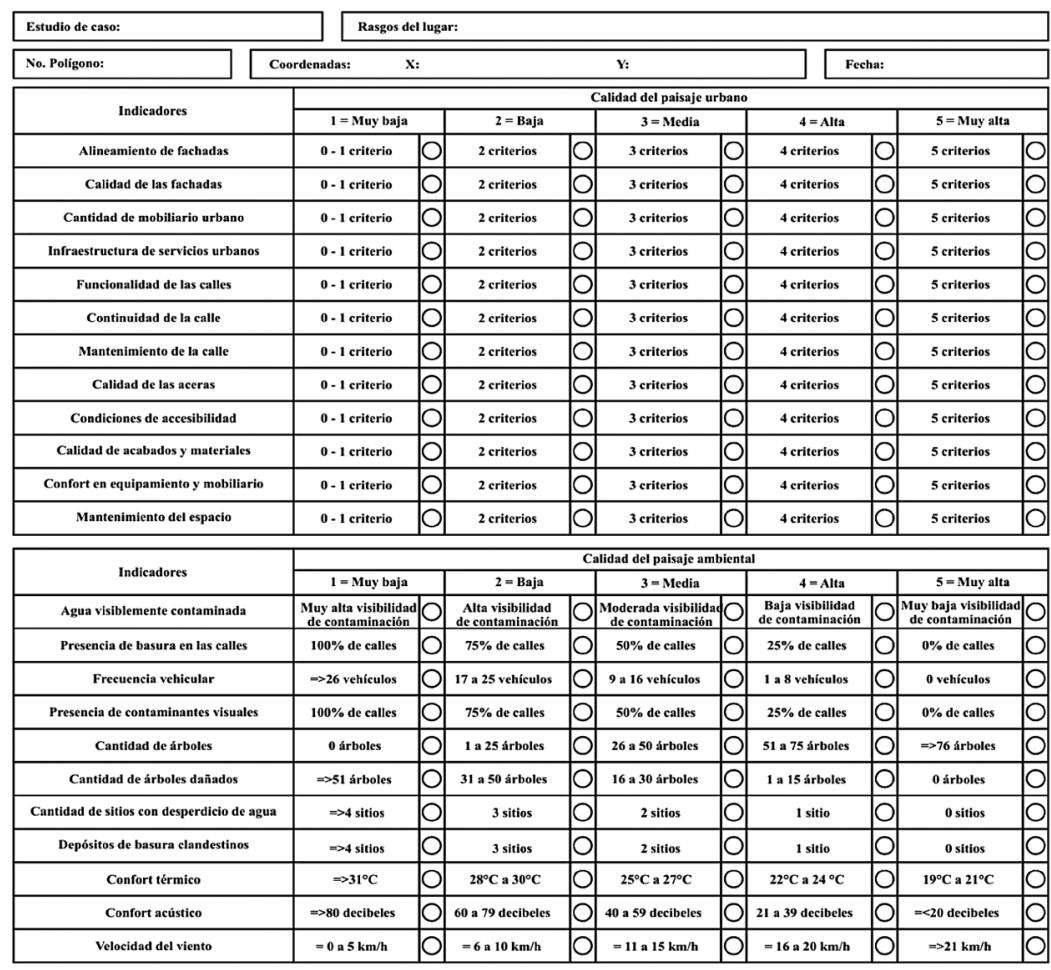

Observaciones

Fuente: elaboración propia con base en recorridos de campo. 


\section{Fuentes consultadas}

Aguilera-Benavente, Francisco; Valenzuela Montes, Luis Miguel y Botequilha-Leitao, Andre (2011), "Landscape metrics in the analysis of urban land use patterns: A case study in a Spanish metropolitan area", Landscape and Urban Planning, 99 (3-4), Ámsterdam, Elsevier, pp. 226-238, doi: 10.1016/j.landurbplan.2010.10.004

Andersson, Erik (2006), "Urban landscapes and sustainable cities”, Ecology and society, 11 (1), Nueva Escocia, Resilience Alliance, pp. 1-7, doi: $10.5751 /$ es-01639-110134

Barrasa García, Sara (2013), "Valoración de la calidad estética de los paisajes de La Habana (Cuba) con métodos de participación social”, Estudios Geográficos, 74 (274), Madrid, Instituto de Economía, Geografía y Demografía, Consejo Superior de Investigaciones Científicas, pp. 45-66, doi: 10.3989/estgeogr.201302

Briceño Avila, Morella y Gil, Beatriz (2003), "Calidad ambiental de la imagen urbana: sectores La Parroquia, Alto Chama, Carrizal, Los Curos, Zona Industrial y barrios La Candelaria y San Buenaventura de la ciudad de Mérida-Venezuela", Fermentum. Revista Venezolana de Sociología y Antropología, 13 (38), Mérida, Universidad de los Andes, pp. 445-482.

Briceño Avila, Morella; Owen de Contreras, Mary Elena y Contreras Miranda, Wilver (2011), "Propuesta de un sistema de indicadores para evaluar la calidad visual del paisaje urbano”, Ecodiseño \& Sostenibilidad, 3 (1), Mérida, Universidad de los Andes/Universidad Politécnica de Valencia, pp. 65-104.

Bürgi, Matthias; Verburg, Peter; Kuemmerle, Tobias y Plieninger, Tobias (2017), "Analyzing dynamics and values of cultural landscapes", Landscape Ecology, 32 (11), Londres, Springer Nature, pp. 20772081, doi: 10.1007/210980-017-0573-0

Buzai, Gustavo D. (2011), "Modelos de localización-asignación aplicados a servicios públicos urbanos: análisis espacial de Centros de Atención Primaria de Salud (CAPS) en la ciudad de Luján, Argentina", Cuadernos de Geografía: Revista Colombiana de Geografí, 20 (2), Bogotá, Universidad Nacional de Colombia, pp. 111-123, doi: 10.15446/rcdg.v20n2.27184 
Buzai, Gustavo D. (2015), "Potencialidad de la metodología de evaluación multicriterio aplicada con sistemas de información geográfica", en Manuel Fuenzalida, Gustavo D. Buzai, Antonio Moreno Jiménez y Armando García de León Loza, Geografía, geotecnología y análisis espacial: tendencias, métodos y aplicaciones, Santiago de Chile, Editorial Triángulo, pp. 99-111.

Cabrerizo Sanz, Casilda (2013), "Ciudad y territorio en clave de paisaje urbano contemporáneo en España y México”, Cuadernos de Vivienda y Urbanismo, 3 (6), Bogotá, Pontificia Universidad Javeriana, pp. 182-195.

Castro, Sergio; Guerreo-Leiva, Nicole; Bolados, Maximiliano y Figueroa, Javier (2018), "Riqueza y distribución de la flora urbana de Santiago de Chile: una aproximación basada en interpolación IDW", Caderno de Pesquisa, 30 (1), Santa Cruz do Sul, Universidade de Santa Cruz do Sul, pp. 41-54, doi: 10.17058/cp.v30i1.12198

Creative Decisions Foundation (2019), "Software Super Decisions", versión 2.10, Pittsburgh, Creative Decisions Foundation.

Ellard, Colin (2015), Places of the heart. The psychogeography of everyday life, Nueva York, Bellevue Literary Press.

ESRI (Economic and Social Resarch Institute) (2013), "Software ArcGis", versión 10.0, Nueva York, ESRI.

Folch, Ramon y Bru, Josepa (2017), Ambiente, territorio y paisaje. Valores y valoraciones, Barcelona-Madrid, Aquae Fundación/Editorial Barcino.

Fuenzalida, Manuel (2015), "Interpolación espacial con sistemas de información geográfica”, en Manuel Fuenzalida, Gustavo Buzai, Antonio Moreno Jiménez y Armando García de León Loza, Geografía, geotecnología y análisis espacial: tendencias, métodos y aplicaciones, Santiago de Chile, Editorial Triángulo, pp. 86-98.

Fürstenau Togashi, Henrique (2009), "Interpretação da paisagem: uma tarefa interdisciplinar", Cuadernos de Geografía: Revista Colombiana de Geografía, núm. 18, Bogotá, Universidad Nacional de Colombia, pp. 71-81, doi: 10.15446/rcdg.n18.13021 
Gailing, Ludger y Leibenath, Markus (2015), "The social construction of landscapes: Two theoretical lenses and their empirical applications", Landscape Research, 40 (2), Didcot, Taylor \& Francis Ltd., pp. 123-138, doi: 10.1080/01426397.2013.775233

Galindo, María Paz y Corraliza, José Antonio (2000), “Environmental aesthetics and psychological wellbeing: relationships between preference judgements for urban landscapes and other relevant affective responses", Psychology in Spain, 4 (1), Madrid, Colegio Oficial de Psicólogos, pp. 13-27.

García, Humberto (2016), "Valoración del paisaje para la planeación territorial de la microcuenca San José el Alto, Querétaro", tesis de maestría, Universidad Autónoma de Querétaro, Querétaro.

Gavrilidis, Athanasios; Ciocanea, Cristiana-Maria; Razvan Nita, Mihai; Onose, Diana Andreea y Năstase, Irina Iulia (2016), "Urban Landscape Quality Index-planning tool for evaluating urban landscape and improving the quality of life", Procedia Environmental Sciences, vol. 32, Ámsterdam, Elsevier, pp. 155-167, doi: 10.1016/j.proenv.2016.03.020

Göbel, Christof A. (2015), "Una visión alemana de los modelos de ciudad. El caso de Querétaro", Revista Gremium, 2 (4), Ciudad de México, Universidad Autónoma Metropolitana, pp. 47-60.

Gobierno Municipal de Querétaro (2008), "Plan Parcial de Desarrollo Urbano para la Delegación Santa Rosa Jáuregui”, Querétaro, Gobierno de Querétaro, <http://www.ordenjuridico.gob.mx/ Estatal/QUERETARO/Municipios/Queretaro/6plan.pdf>, 25 de febrero de 2018.

Godínez Flores, Juan Manuel y Alvarez-Castañon, Lorena del Carmen (2017), "Política de innovación y desarrollo industrial: retos y deudas sociales en Querétaro, México", Revista Vértice Universitario, núm. 76, Hermosillo, Universidad de Sonora, pp. 7-17.

Guzman, Paloma; Pereira Roders, A. R. y Colenbrander, Bernard (2017), "Measuring links between cultural heritage management and sustainable urban development: An overview of global monitoring tools", Cities, 60 (A), Ámsterdam, Elsevier, pp. 192-201, doi: 10.1016/j.cities.2016.09.005 
Hanafi-Bojd, Ahmad Ali; Vatandoost, Hassan; Oshaghi, Mohammad; Charrahy, Zabihollah; Haghdoost, Aliakbar; Zamani, Ghasem; Abedi, Farshid; Sedaghat, Mohammad; Soltani, Maziar; Shahi, Manisha y Raeisi, Ahmad (2012), "Spatial analysis and mapping of malaria risk in an endemic area, south of Iran: A GIS based decision making for planning of control”, Acta Tropica, 122 (1), Ámsterdam, Elsevier, pp. 132-137, doi: 10.1016/j.actatropica.2012.01.003

Hernández-Guerrero, Juan (2015), "Valoración visual de la calidad ambiental del área urbana de Querétaro, México: la compleja sencillez de valorar el entorno urbano", Revista de Geografia Norte Grande, núm. 61, Santiago de Chile, Pontificia Universidad Católica de Chile, pp. 45-64, doi: 10.4067/S0718-34022 015000200004

Hernández-Guerrero, Juan y Osorno Sánchez, Tamara (2018), “Diferencias ambientales en el paisaje urbano de la ciudad de Querétaro, México: caso de estudio Juriquilla y Santa Rosa Jáuregui", Revista de Geografía Norte Grande, núm. 71, Santiago de Chile, Pontificia Universidad Católica de Chile, pp. 147-166, doi: 10.4067/ S0718-34022018000300147

Hernández-Guerrero, Juan; Luna, Hugo; Navarrete, Alma y Martínez, Griselda (2016), "Expansión urbana y precariedad habitacional en el área urbana del municipio de Querétaro, México: 19802010”, en Antonio Vieyra, Yadira Méndez y Juan Hernández Guerrero (coords.), Procesos urbanos, pobreza y ambiente. Implicaciones en ciudades medias y megaciudades, Morelia, Centro de Investigaciones en Geografía Ambiental/Universidad Nacional Autónoma de México, pp. 109-124.

Hofmann, Mathias; Westermann, Janneke; Kowarik, Ingo y Van der Meer, Elke (2012), "Perceptions of parks and urban derelict land by landscape planners and residents", Urban Forestry \& Urban Greening, 11 (3), Ámsterdam, Elsevier, pp. 303-312, doi: 10.1016/j.ufug.2012.04.001

Inegi (Instituto Nacional de Estadística y Geografía) (2017), Anuario estadístico y geográfico de Querétaro, Aguascalientes, Inegi, <http:// www.datatur.sectur.gob.mx/ITxEF_Docs/QRO_ANUARIO_ PDF $>, 25$ de marzo de 2018. 
Inegi (Instituto Nacional de Estadística y Geografía) (2015a), Conteo de Población y Vivienda 2015, Aguascalientes, Inegi, <https://www. inegi.org.mx/programas/intercensal/2015/default. html\#Tabulados>, 16 de febrero de 2018.

Inegi (Instituto Nacional de Estadística y Geografía) (2015b), Conjunto de datos vectoriales y toponímicos, escala 1:50,000, Aguascalientes, Inegi, <https://www.inegi.org.mx/app/mapas/default.html?t=015 0001000000000\&ag=22>, 11 de septiembre de 2019.

Inegi (Instituto Nacional de Estadística y Geografía) (1990), Censo de Población y Vivienda 1990, Aguascalientes, Inegi, < https://www. inegi.org.mx/programas/ccpv/1990/>, 08 de diciembre de 2017.

Jusidman de Bialostozky, Clara; Camas, Francisco; Carreón, Ingrith y Marín, Osiris (2016), El crecimiento urbano y las violencias en México, Ciudad de México, Comisión Económica para América Latina y el Caribe.

Kaliraj, Seenipandi; Chandrasekar, Nainarpandian y Magesh, Sivan (2015), "Evaluation of multiple environmental factors for sitespecific groundwater recharge structures in the Vaigai River upper basin, Tamil Nadu, India, using GIS-based weighted overlay analysis", Environmental Earth Sciences, 74 (5), Londres, Springer Nature, pp. 4355-4380, doi: 10.1007/s12665-015-4384-9

Karmanov, Dmitri y Hamel, Ronald (2008), "Assessing the restorative potential of contemporary urban environment(s): Beyond the nature versus urban dichotomy", Lanscape and urban planning, 86 (2), Ámsterdam, Elsevier, pp. 115-125, doi: 10.1016/j.landurbplan.2008.01.004

Keshtkaran, Reza; Habibi, Amin y Sharif, Hamidreza (2017), “Aesthetic preferences for visual quality of urban landscape in Derak HighRise Buildings (Shiraz)", Journal of Sustainable Development, 10 (5), Richmond Hill, Canadian Center of Science and Education, pp. 94-106, doi:10.5539/jsd.v10n5p94

Lalana Soto, José Luis (2011), “El paisaje urbano histórico: Modas, paradigmas y olvidos", Ciudades, 14 (1), Valladolid, Instituto Universitario de Urbanística de la Universidad de Valladolid, pp. 15-38. 
Liu, Lun; Silva, Elisabete; Wu, Chunyang y Wang, Hui (2017), “A machine learning-based method for the large-scale evaluation of the qualities of the urban environment", Computers, environment and urban systems, vol. 65, Ámsterdam, Elsevier, pp. 113-125, doi:10.1016/j.compenvurbsys.201 7.06.003

Maderuelo, Javier (2005), El paisaje. Génesis de un concepto, Madrid, Abada Editores.

Maderuelo, Javier (2010), “El paisaje urbano”, Estudios Geográficos, 71 (269), Madrid, Instituto de Economía, Geografía y Demografía/ Consejo Superior de Investigaciones Científicas, pp. 575-600, doi: 10.3989/estgeogr.201019

Matsuoka, Rodney y Kaplan, Rachel (2008), "People needs in the urban landscape: analysis of landscape and urban planning contributions", Landscape and urban planning, 84 (1), Ámsterdam, Elsevier, pp. 7-19, doi: 10.1016/j.landurbplan.2007.09.009

Medina Muro, María (2010), "La práctica del paisajismo", Estudios Geográficos, 71 (269), Madrid, Instituto de Economía, Geografía y Demografía, Consejo Superior de Investigaciones Científicas, pp. 633-658, doi: 10.3989/estgeogr.201021

Muñoz-Pedreros, Andrés (2004), "La evaluación del paisaje: una herramienta de gestión ambiental", Revista Chilena de Historia Natural, 77 (1), Santiago de Chile, Sociedad de Biología de Chile, pp. 139-156.

Nogué, Joan (2010), "El paisaje en la ordenación del territorio. La experiencia del Observatorio del Paisaje de Cataluña”, Estudios Geográficos, 71 (269), Madrid, Instituto de Economía, Geografía y Demografía/Consejo Superior de Investigaciones Científicas, pp. 415-448, doi: 10.3989/estgeogr.201014

Nogué, Joan; de-San-Eugenio-Vela, Jordi y Sala, Pere (2019), "La implementación de indicadores de lo intangible para catalogar el paisaje percibido. El caso del Observatorio del Paisaje de Cataluña”, Revista de Geografía Norte Grande, núm. 72, Santiago de Chile, Pontificia Universidad Católica de Chile, pp. 75-91, doi: 10.4067/ S0718-34022019000100075 
Pascual González, Aylín y Peña Díaz, Jorge (2012), "Espacios abiertos de uso público", Arquitectura y Urbanismo, 33 (1), La Habana, Instituto Superior Politécnico José Antonio Echeverría, pp. 25-42.

Pasimeni, Maria Rita; De Marco, Antonella; Petrosillo, Irene; Aretano, Roberta; Semeraro, Teodoro; Zaccarelli, Nicola y Zurlini, Giovanni (2012), "New perspectives and approaches in social-ecological landscape evaluation", Plurimondi, núm. 10, Bari, European Colloquium on Theoretical and Quantitative Geography, pp. 109-118.

Pérez Hernández, Edmundo (2000), "Paisaje urbano en nuestras ciudades”, Bitácora urbano-territorial, 4 (1), Bogotá, Universidad Nacional de Colombia, pp. 33-37.

PNUMA (Programa de las Naciones Unidas para el Medio Ambiente) (2008), Perspectivas del medio ambiente urbano. GEO Zona Metropolitana de Querétaro, Querétaro, PNUMA.

Stewart, Thomas; Middleton, Paulette y Ely, Daniel (1983), "Urban visual air quality judgements: reliability and validity", Journal of Environmental Psychology, 3 (2), Ámsterdam, Elsevier, pp. 129-145, doi: $10.1016 / S 0272-4944(05) 80152-5$

Taylor, Ken (2016), “The historic urban landscape paradigm and cities as cultural landscapes. Challenging orthodoxy in urban conservation", Landscape Research, 41 (4), Didcot, Taylor \& Francis, pp. 471-480, doi: 10.1080/01426397.2016.1156066

Urquijo, Pedro S. y Barrera-Bassols, Narciso (2009), "Historia y paisaje. Explorando un concepto geográfico monista”, Andamios, 5 (10), Ciudad de México, Universidad Autónoma de la Ciudad de México, pp. 227-252.

Urquijo, Pedro S. y Bocco, Gerardo (2011), "Los estudios de paisaje y su importancia en México, 1970-2010", Journal of Latin American Geography, 10 (2), Austin, Universidad of Texas, pp. 37-63.

Varatharajan, Ramachandran; Manogaran, Gunasekaran; Priyan, Malarvizhi; Balas, Valentina y Barna, Cornel (2018), "Visual analysis of geospatial habitat suitability model based on inverse distance weighting with paired comparison analysis", Multimedia Tools 
and Applications, 77 (14), Londres, Springer Nature, pp. 1757317593, doi: 10.1007/s11042-017-4768-9

Wu, Jianguo (2010), "Urban sustainability: an inevitable goal of landscape research", Landscape Ecology, 25 (1), Londres, Springer Nature, pp. 1-4, doi: 10.1007/s10980-009-9444-7

Recibido: 24 de febrero de 2020. Reenviado: 21 de mayo de 2020. Aceptado: 14 de julio de 2020.

Juan Alfredo Hernández Guerrero. Doctor en Geografía por la Universidad Nacional Autónoma de México. Actualmente es profesor-investigador de tiempo completo en la Universidad Autónoma de Querétaro y coordinador de la Maestría en Gestión Integrada de Cuencas. Miembro del Sistema Nacional de Investigadores, nivel I. Sus líneas de investigación son: geografía del riesgo, análisis urbano-regional y gestión de cuencas hidrográficas. Entre sus últimas publicaciones se encuentran: como coautor, "Ciclo hidrosocial y acceso al agua en la periferia de la ciudad de Morelia, México: Estudio de caso en La Aldea", Revista Geográfica de América Central, 64 (1), Heredia, Universidad Nacional de Costa Rica, pp. 269-297 (2020); "Diferencias ambientales en el paisaje urbano de la ciudad de Querétaro, México: Caso de estudio Juriquilla y Santa Rosa Jáuregui", Revista de Geografía Norte Grande, núm. 71, Santiago de Chile, Pontificia Universidad Católica de Chile, Instituto de Geografía, pp. 147166 (2018); como editor, Gestión integrada de cuencas. Una experiencia conjunta en el volcán El Zamorano, Querétaro, Universidad Autónoma de Querétaro (2019). 